\title{
Heteromeric Assembly of Truncated Neuronal Kv7 Channels: Implications for Neurologic Disease and Pharmacotherapy
}

\author{
Jingru Li, Jasmine Maghera, Shawn M. Lamothe, Elysa J. Marco, and Harley T. Kurata \\ Department of Pharmacology, Alberta Diabetes Institute, University of Alberta, Edmonton, Alberta, Canada (J.L., J.M., S.M.L., \\ H.T.K.) and Department of Neurodevelopmental Medicine, Cortica Healthcare, San Rafael, California (E.J.M.)
}

Received February 4, 2020; accepted June 11, 2020

\begin{abstract}
Neuronal voltage-gated potassium channels (Kv) are critical regulators of electrical activity in the central nervous system. Mutations in the KCNQ (Kv7) ion channel family are linked to epilepsy and neurodevelopmental disorders. These channels underlie the neuronal "M-current" and cluster in the axon initial segment to regulate the firing of action potentials. There is general consensus that KCNQ channel assembly and heteromerization are controlled by C-terminal helices. We identified a pediatric patient with neurodevelopmental disability, including autism traits, inattention and hyperactivity, and ataxia, who carries a de novo frameshift mutation in KCNQ3 (KCNQ3-FS534), leading to truncation of $\sim 300$ amino acids in the $C$ terminus. We investigated possible molecular mechanisms of channel dysfunction, including haplo-insufficiency or a dominant-negative effect caused by the assembly of truncated KCNQ3 and functional KCNQ2 subunits. We also used a recently recognized property of the KCNQ2-specific activator ICA-069673 to identify assembly of heteromeric channels. ICA-069673 exhibits a functional signature that depends on the subunit composition of KCNQ2/3 channels, allowing us to determine whether truncated KCNQ3 subunits can assemble with KCNQ2. Our findings demonstrate that although
\end{abstract}

the KCNQ3-FS534 mutant does not generate functional channels on its own, large C-terminal truncations of KCNQ3 (including the KCNQ3-FS534 mutation) assemble efficiently with KCNQ2 but fail to promote or stabilize KCNQ2/KCNQ3 heteromeric channel expression. Therefore, the frequent assumption that pathologies linked to KCNQ3 truncations arise from haplo-insufficiency should be reconsidered in some cases. Subtype-specific channel activators like ICA-069673 are a reliable tool to identify heteromeric assembly of KCNQ2 and KCNQ3.

\section{SIGNIFICANCE STATEMENT}

Mutations that truncate the $\mathrm{C}$ terminus of neuronal $\mathrm{Kv} 7 / \mathrm{KCNQ}$ channels are linked to a spectrum of seizure disorders. One role of the multifunctional KCNQ C terminus is to mediate subtypespecific assembly of heteromeric KCNQ channels. This study describes the use of a subtype-specific Kv7 activator to assess assembly of heteromeric KCNQ2/KCNQ3 (Kv7.2/Kv7.3) channels and demonstrates that large disease-linked and experimentally generated C-terminal truncated $\mathrm{KCNQ} 3$ mutants retain the ability to assemble with KCNQ2.

\section{Introduction}

KCNQ (Kv7) channels are voltage-gated potassium channels with physiologic roles in the regulation of cardiac rhythm, neuronal activity, peripheral smooth muscle contractions, and endocrine function. KCNQ2 (Kv7.2) and KCNQ3 (Kv7.3) form heteromeric channels throughout the central nervous system and are the primary constituents of M-channels (Wang et al., 1998). These conduct potassium currents essential for the control of threshold and burst firing properties of neurons (Adams and Brown, 1982; Cooper et al., 2000; Shapiro et al., 2000; Hill et al., 2008), and mutations in both KCNQ2 and KCNQ3 have been identified in a spectrum of epilepsy conditions that manifest in infancy or early childhood (Biervert et al., 1998; Charlier et al., 1998; Singh et al., 1998). M-channels are

This work was funded by the Canadian Institutes of Health Research [Grant MOP-97988] to H.T.K. S.M.L. was supported by a Rowland and Muriel Haryett Fellowship, University of Alberta Neuroscience and Mental Health Institute. J.L. was supported by a Canadian Institutes of Health Research CGS-M award. H.T.K. was supported by a Canadian Institutes of Health Early Career Investigator award and salary support from the Alberta Diabetes Institute.

https://doi.org/10.1124/mol.120.119644. voltage-gated and also exhibit prominent physiologic regulation by phosphatidylinositol-4,5-bisphosphate (Zhang et al., 2003; Suh et al., 2006). This property was first recognized as a sensitivity to muscarine, now understood to arise from phosphatidylinositol-4,5-bisphosphate depletion after M1 acetylcholine receptor-mediated activation of $\mathrm{G}_{\mathrm{q} / 11}$ and phospholipase C (Brown and Adams, 1980). KCNQ3 mutations are most commonly associated with benign familial neonatal epilepsy, which is typically responsive to conventional antiepileptic drugs and resolves within the first few years of life with no structural or cognitive impairment (Miceli et al., 1993b; Maljevic and Lerche, 2014). Although KCNQ2 commonly forms heteromeric channels with KCNQ3, KCNQ2 channels are more commonly linked to far more severe neurologic deficits, including pharmacoresistant seizures and global neurodevelopmental delay (Miceli et al., 1993a; Weckhuysen et al., 2012; Lerche et al., 2013; Maljevic and Lerche, 2014; Millichap et al., 2016).

Assembly of KCNQ2 and KCNQ3 generates heteromeric channels with biophysical hallmarks distinct from either subunit alone, including unique voltage dependence, enhanced 
current expression, and altered pharmacological sensitivity (Wang et al., 1998; Shapiro et al., 2000). It is generally thought that the $\mathrm{C}$ terminus of KCNQ channels governs subtypespecific channel assembly. For example, KCNQ1 and KCNQ3 channels are not compatible and do not generate functional heteromers, but this exclusion can be overcome by the chimeric swapping of C-terminal regions (Schmitt et al., 2000; Maljevic et al., 2003; Schwake et al., 2003, 2006). The C terminus contains segments with high probabilities for the formation of coiled-coiled domains thought to contribute to channel assembly and influence subunit specificity (Howard et al., 2007). However, there remains uncertainty regarding how subtype-specific assembly of KCNQ2-5 subtypes is encoded by this region.

ClinVar and RIKEE (www.rikee.org) (Millichap et al., 2016) databases report a variety of KCNQ2 and KCNQ3 frameshift/ truncation mutations (locations are summarized visually in Fig. 1A). KCNQ2 truncations often cause severe phenotypes (e.g., epileptic encephalopathy, red symbols in Fig. 1A) compared with similar KCNQ3 truncations (e.g., benign familial epileptic seizures, green symbols in Fig. 1A). In these cases of KCNQ-related disease, it may be unclear

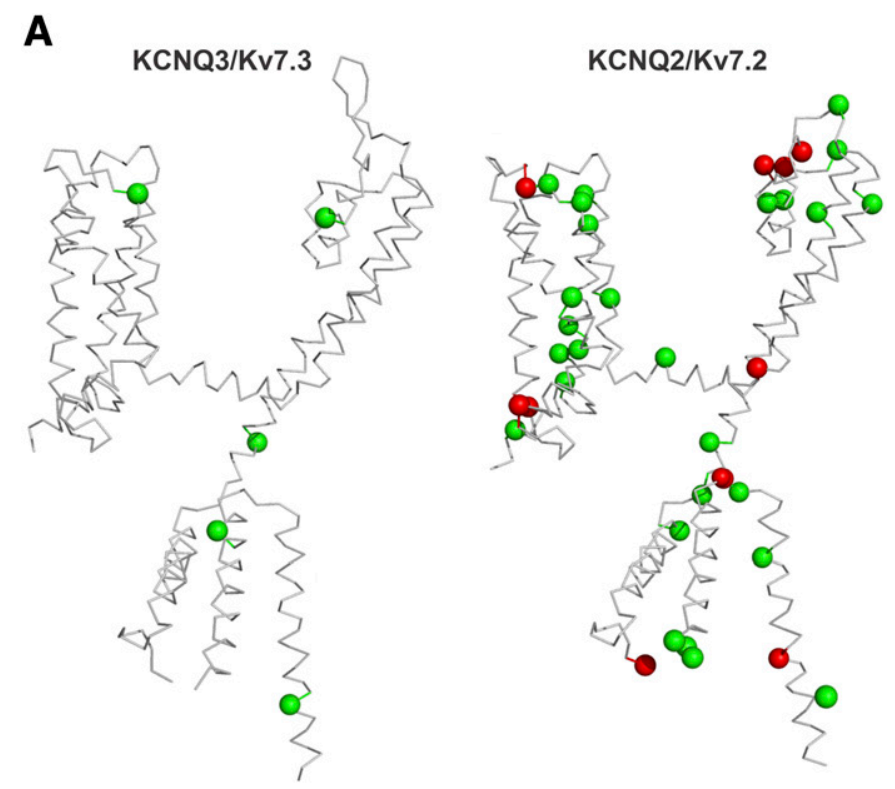

B

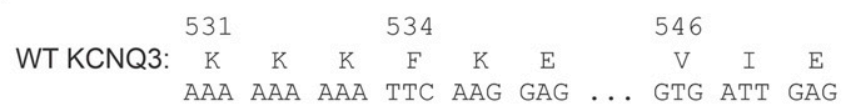

$\begin{array}{ccccccccccc}\text { FS -534: } & \text { K } & K & K & I & Q & \text { G } & & \text { C } & \text { D } & - \\ & \text { AAA } & \text { AAA } & \text { AAA } & \text { ATT } & \text { CAA } & \text { GGA } & \ldots & \text { TGT } & \text { GAT } & \text { TGA }\end{array}$

Fig. 1. Truncation and frameshift mutations of KCNQ2 and KCNQ3. (A) Locations of frameshift and truncation mutations in KCNQ2 and KCNQ3 reported on ClinVar or RIKEE databases. Mutations associated with benign familial neonatal epilepsy are highlighted in green, whereas mutations associated with epileptic encephalopathy or other severe syndromes are highlighted in red. (B) A de novo adenine insertion in KCNQ3 was identified in a patient exhibiting traits of autism and neurodevelopmental delay. The patient sequence is presented along with the reference WT KCNQ3 sequence. The frameshift causes premature truncation at amino acid position 548, leading to the modification of 14 amino acids and truncation of 324 amino acids. whether loss of function arises from haplo-insufficiency versus dominant-negative effects from assembly of mutated subunits with normal subunits. That is, truncation of one copy of KCNQ2 or KCNQ3 may reduce the number of functional subunits required for normal M-channel activity, or alternatively, truncated subunits may assemble with wildtype subunits and suppress function in a dominant-negative manner.

In a recent study, Lauritano et al. (2019) identified a homozygous KCNQ3 truncation at amino acid position 534 (KCNQ3-FS534) in a patient with intellectual disability and pharmaco-dependent epilepsy (Lauritano et al., 2019). We simultaneously investigated a patient with an identical mutation (but heterozygous and de novo) and used this as a starting point to study the effects of KCNQ channel truncations on heteromeric KCNQ2/KCNQ3 channel assembly and function. We devised a pharmacological strategy to assess heteromeric channel assembly based on subtype-specific actions of certain voltage sensor-targeted $\mathrm{KCNQ} / \mathrm{Kv} 7$ activator drugs (Padilla et al., 2009; Wang et al., 2017, 2018a). ICA069673 causes pronounced deceleration of deactivation of homotetrameric KCNQ2 channels, but these effects are largely attenuated when KCNQ2 and KCNQ3 are coassembled (Wang et al., 2018a,b). We used this assay to test heteromeric assembly of KCNQ2 with the disease-linked KCNQ3 truncation mutant, along with a series of artificial KCNQ3 truncation mutants. Pharmacological characterization offers unambiguous evidence that large truncations of KCNQ3 retain the ability to assemble and form heterotetrameric channels with KCNQ2, similar to wild-type (WT) KCNQ3. These findings suggest that heteromeric assembly may occur in patients and can contribute to a dominant-negative effect.

\section{Materials and Methods}

Molecular Biology. Wild-type and mutant KCNQ2 and KCNQ3 channel cDNA constructs were expressed using the pTLN expression vector. The KCNQ3[A315T] and KCNQ3[A315T]-FS534 were expressed using the pSRC5 vector (gifts from M. Taglialatela, University of Molise, Campobasso, Italy, and T. Jentsch, Leibniz-Institut fur Molekulare Pharmakologie, Berlin), where the A315T mutation is used to enhance homomeric KCNQ3 expression (Etxeberria et al., 2004; Gómez-Posada et al., 2010). KCNQ3-FS534 mutant cDNAs were generated on WT KCNQ3 or KCNQ3[A315T] backgrounds using a two-step overlapping polymerase chain reaction (PCR) approach. Artificial KCNQ3 truncation mutant cDNAs were generated on WT KCNQ3 by introducing stop codons in a one-step PCR approach. All cDNAs were verified via Sanger sequencing. cDNAs were linearized using MluI for pTLN(KCNQ2), HpaI for pTLN(KCNQ3), and ApaLI for pSRC5(KCNQ3[A315T]) and ethanol precipitated to concentrate templates for RNA transcription. cRNA was transcribed from concentrated linear cDNAs using the Ambion mMessage mMachine kits (Thermo Fisher Scientific, Waltham, MA): SP6 kit to transcribe from pTLN and T7 kit to transcribe from pSRC5.

Mutagenic primers for the KCNQ3-FS534 mutation were as follows: FS534 forward: 5'-CCGTCTCTATAAAAAAAAAATTCAAGGAGAC3' (on WT KCNQ3 and KCNQ3[A315T] backgrounds); FS534 reverse: 5'-GTCTCCTTGAATTTTTTTTTTATAGAGACGG-3' (on WT KCNQ3 and KCNQ3[A315T] backgrounds).

After generation of a KCNQ3 PCR fragment containing the FS534 mutation, the fragment was inserted in the KCNQ3[A315T] pSRC5 plasmid using NotI and EcoRI digestion and ligation. FS534 generated on the WT KCNQ3 background was inserted in the KCNQ3-pTLN plasmid using NotI and KpnI digestion and ligation. 
Artificial truncations of WT KCNQ3 were generated by PCR reactions using a common forward primer (Q3Forward) and various reverse primers as follows: Q3Forward: 5'-GGCGACGTGGAGCAA GTCACC- 3 '; $\triangle$ C503 reverse: 5'-GCGCGGTACCCTACTCAAAGTGCT TCTGACG-3'; $\Delta$ C340 reverse: 5'-GCGCGGTACCCTAATAGAGAC GGAATTGTAG-3'; $\Delta$ C301 reverse: 5'-GCGCGGTACCCTAAATCA TATCTATTCTCGTC-3'; $\Delta$ C42 reverse: 5'-GCGCGGTACCCTAGC TTCTCCCTCATCCAGC-3'.

PCR fragments were cloned into the KCNQ3-pTLN plasmid using NotI and KpnI digestion and ligation. All constructs were confirmed using restriction digestion and Sanger sequencing (The Applied Genomics Core, University of Alberta, Edmonton, AB, Canada).

Oocyte Preparation and Injection. Stage V-VI Xenopus laevis oocytes were obtained surgically and were defolliculated for 22.5 hours in a $3 \mathrm{mg} / \mathrm{ml}$ type IV collagenase saline solution. Oocytes were injected with 25-50 ng of total channel cRNA for homomeric injections and heteromeric KCNQ2 + KCNQ3 coinjections, and the total mRNA injected within each experiment was controlled. After injection, oocytes were incubated for 24-48 hours before recording at $17^{\circ} \mathrm{C}$ in OR3 medium: $500 \mathrm{ml}$ Liebovitz's L-15 medium, $15 \mathrm{mM}$ HEPES, $1 \mathrm{mM}$ L-glutamine, $0.5 \mathrm{mM}$ gentamicin, distilled and deionized water to 11 , and adjusted to $\mathrm{pH}$ 7.4. Oocyte preparation and RNA injection were done under the approval of University of Alberta Animal Care protocol AUP00001752.

Two-Electrode Voltage Clamp. Voltage-clamped potassium currents were recorded in a modified Ringer's solution (in millimoles): $116 \mathrm{NaCl}, 2 \mathrm{KCl}, 1 \mathrm{MgCl}_{2}, 0.5 \mathrm{CaCl}_{2}, 5 \mathrm{HEPES}$, and adjusted to $\mathrm{pH}$ 7.4. Voltage clamps were done using an OC-725C voltage clamp (Warner, Hamden, CT). Glass microelectrodes were pulled using a Sutter Micropipette puller (Sutter Instrument, Novato, CA), backfilled using $3 \mathrm{M} \mathrm{KCl}$, and had resistances of $0.1-1 \mathrm{M} \Omega$. Data were filtered at $5 \mathrm{kHz}$ and digitized at $10 \mathrm{kHz}$ using a Digidata 1440A (Molecular Devices, Sunnyvale, CA) controlled by pClamp 10 software (Molecular Devices). Retigabine (RTG) was purchased from Toronto Research Chemicals (North York, ON, Canada), ICA-069673 (ICA73) was purchased from Tocris (Bristol, United Kingdom), and both were stored as $100 \mathrm{mM}$ stocks in DMSO and diluted to working concentrations each experimental day in the same modified Ringer's solution used for recording. Oocytes were incubated in RTG for 10 minutes or in ICA069673 for 3-4 minutes prior to two-electrode voltage-clamp recordings in the drug solutions.

KCNQ Channel Molecular Models. Molecular models of KCNQ2 and KCNQ3 were generated using the online SWISSMODEL tool (Waterhouse et al., 2018), using the 2017 KCNQ1 cryoelectron microscopy structure as a guide (Sun and MacKinnon, 2017). These models are for illustration only and have not undergone refinement or development beyond this web-based homology modeling tool.

Data Analysis. Voltage dependence of channel activation was fit using a standard single component Boltzmann equation of the form

$$
G / G \max =1 /\left(1+e^{-\left(V-V_{1 / 2}\right) / k}\right),
$$

where $V_{1 / 2}$ is the voltage where channels exhibit half-maximal activation, $k$ is the slope factor reflecting the voltage range over which an $e$-fold change in the open probability $\left(\mathrm{P}_{\mathrm{o}}\right)$ is observed, and G/Gmax is the proportion of channels in the open and conducting state. Fractional instantaneous currents were determined as the ratio of the instantaneous current divided by the maximum current in the presence or absence of ICA-069673. Varied statistical tests were used depending on the context and experimental design and are described in individual figure legends.

Boltzmann fits to conductance-voltage relationships were fit by least squares minimization of the single component Boltzmann equation to the data, using the Solver algorithm in Microsoft Excel (Microsoft, Redmond, WA). Beyond this analysis, no additional models were assumed or applied in our study. Box plots were generated with Sigmaplot (Systat Software, San Jose, CA). The box depicts the median and the 25 th-75th percentile range, and the whiskers depict the 10 th-90th percentile range.

\section{Results}

Clinical Features of a Heterozygous KCNQ3-FS534 Patient. Our initial observation of the KCNQ3-FS534 frameshift mutation was made in an 8-year-old male patient at first evaluation at an academic center's child neurology division. He meets Diagnostic and Statistical Manual of Mental Disorders, 5th Edition-based criteria for autism spectrum disorder and attention deficit hyperactivity disorder, combined type, as well as more generally exhibiting global developmental challenges. Developmentally, he showed intact early gross motor milestones at 12 months of age but expressive language delay with sounds but no words at 1 year. At 24 months, he stopped making communicative utterance and social response to name. At 8 years of age, the patient exhibited relatively strong gross motor abilities, including the ability to run, climb, and swim independently, but struggled with fine motor skills with limited writing and ability to dress independently. His expressive language at this time was limited to single words to express needs and wants. At 11 years of age his abilities and performance are greatly impacted by inattention and hyperactivity. He continues to struggle with fine motor control and is able to follow one-step directions. He primarily uses single words and word approximations but can use high yield repetitive phrases for requests. He is using an assisted communication device at school. His sensory processing ability is notable for underresponsivity in the vestibular and proprioceptive domains and overresponsivity visually. He continues to have difficulty with joint attention, orienting to social stimuli, reciprocal interaction, and imitation.

Exome sequencing revealed the presence of a de novo KCNQ3 gene truncation, arising from a frameshift at amino acid position 534 that resulted from a single adenine insertion (within a repetitive polyadenine sequence, Fig. 1B), leading to truncation of the protein at amino acid position 548. A GeneDX array, mitochondrial DNA sequencing, fragile $X$ screening, and chromosome microarray did not reveal other noteworthy findings. No other causative variants were found in disease genes associated with the reported phenotype. This variant is not present in the gnomAD or Exome Variant Server databases and has two entries (including this patient) in the National Center for Biotechnology Information ClinVar database. In terms of family history, the mother exhibited delayed speech onset, and a paternal uncle had been diagnosed with dyslexia and autism spectrum disorder traits. However, neither parent carried the KCNQ3-FS534 mutation, and so this mutation was described as de novo. These findings were deemed clinically significant, but there were no reports of delay with comparable severity as observed in the proband. The patient had no history of seizures; however, there was a staring spell reported at 6 years of age. Overnight video telemetry done at time of genetic diagnosis showed no epileptiform activity with no atypical response to photic stimulation or hyperventilation and intact anteroposterior organization and reactive posterior dominant rhythm of $10 \mathrm{~Hz}$. Sleep architecture was also intact. Magnetic resonance imaging of the brain did not show structural abnormalities or injury.

Relative to KCNQ2, reports of pathologic mutations in KCNQ3 are infrequent and are more often associated with 
benign neonatal or infantile seizures, rather than severe epileptic encephalopathy or neurodevelopmental delay. Figure 1A illustrates a map of the location of KCNQ2 and KCNQ3 frameshift/truncation mutations currently deposited on ClinVar, color coded for severity. It is apparent that truncation mutations scattered throughout both channels have been identified, and reports in KCNQ2 far outweigh KCNQ3 in terms of sheer numbers and severity. There is not a clear pattern that predicts the severity of outcome of a truncation mutation.

A recent report by Lauritano et al. (2019) highlighted the discovery of an identical frameshift, but with a pattern of recessive inheritance in an Italian family (Lauritano et al., 2019). The affected patient was homozygous for this frameshift and exhibited neonatal seizures that were effectively treated with various anticonvulsants (currently sodium valproate monotherapy). Prior to treatment, the patient exhibited speech delay and moderate intellectual disability. However, this patient also exhibited epilepsy, which was not observed in the patient in our study. It was noted that one family member was reported to exhibit mild learning difficulties and transient neonatal seizures, but other heterozygous carriers of this frameshift mutation exhibited normal development and no seizures. In our case, the KCNQ3-FS534 appeared de novo in the child. The penetrance of neurologic traits despite their heterozygous genotype suggests that other genetic factors, or environmental factors encountered in childhood, likely also influence the severity of KCNQ3 mutations. This may not be surprising as these channels likely have shifting patterns of expression and functional importance very early in development.

Functional Characterization of the KCNQ3-FS534 Mutation in X. laevis Oocytes. Although the $\mathrm{C}$ terminus of KCNQ channels has been implicated in many studies as a mediator of channel assembly and subunit specificity (e.g., excluding KCNQ2 or KCNQ3 assembly with KCNQ1), there is not a good understanding of features that drive assembly and maturation of noncardiac KCNQ isoforms or of what may govern subtype specificity among KCNQ2-5. Using the KCNQ3-FS534 mutant as a launching point, we studied the influence of KCNQ3 truncations on assembly of heteromeric M-channels. We first reconstituted the KCNQ3-FS534 mutation in both the WT KCNQ3 and the KCNQ3[A315T] background channels to examine effects on expression and function (the KCNQ3[A315T] background enhances currents $>30$-fold when compared with WT KCNQ3) (Etxeberria et al., 2004; Gómez-Posada et al., 2010). Consistent with the recent description of KCNQ3-FS534 (Lauritano et al., 2019), we observed that the truncated mutant of KCNQ3 leads to a pronounced reduction of total current equivalent to background uninjected levels in $X$. laevis oocytes (Fig. 2). Additionally, the nonselective antiepileptic neuronal KCNQ opener RTG was unable to rescue channel function (Fig. 2, $\mathrm{B}$ and $\mathrm{C}$ ).

Using the KCNQ3-FS534 mutation on a WT KCNQ3 background, we tested the effects of coexpression with KCNQ2 to mimic the native heteromeric composition of M-channels. We assessed current magnitude 48 hours after oocyte injection (Fig. 3). We observed clearly resolvable currents in oocytes expressing WT KCNQ2 homomeric channels, whereas KCNQ3-FS534 channels did not generate any functional current alone (Fig. 3, A and B). Coexpression of WT KCNQ2 and WT KCNQ3 (1:1 ratio of injected mRNA) produced a synergistic increase of total current relative to KCNQ2 or KCNQ3 alone (Fig. 3, A and D). However, the same combination of KCNQ2 and KCNQ3-FS534 failed to recapitulate this enhancement of current (Fig. 3, A and C). We noted that the KCNQ3-FS534 mutant did not suppress channel currents, resulting in currents that are similar in amplitude to KCNQ2 homomers (Fig. 3A). However, this does not clearly distinguish
A
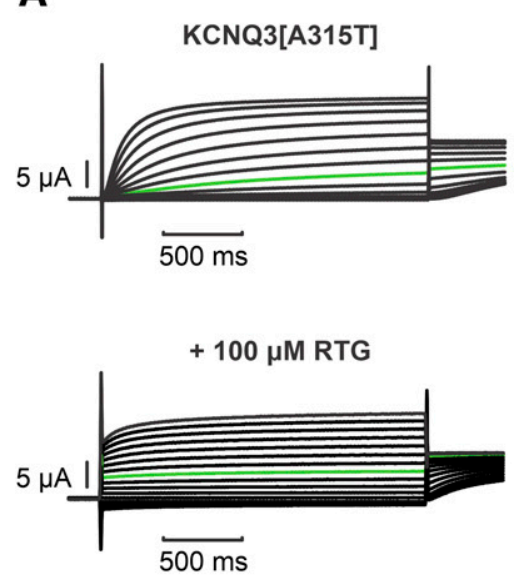

B
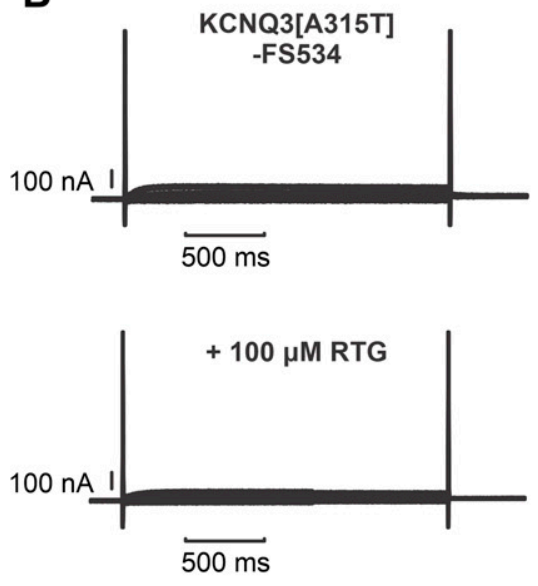

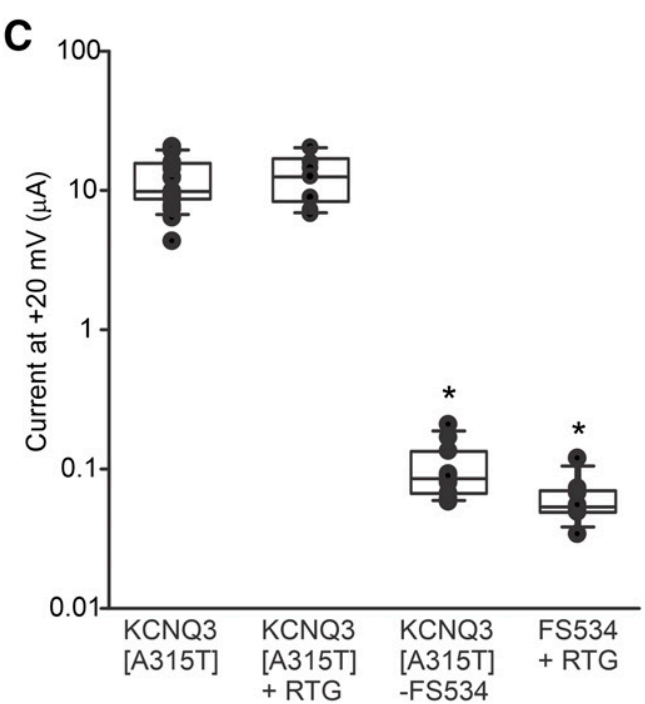

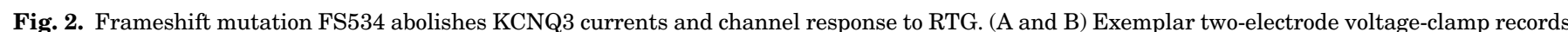

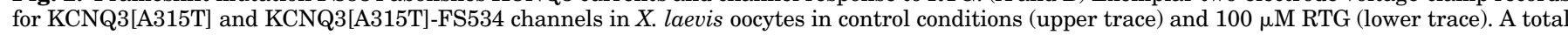

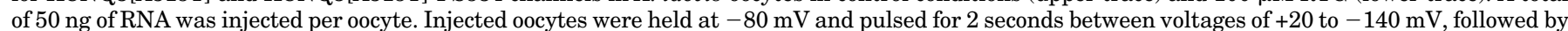

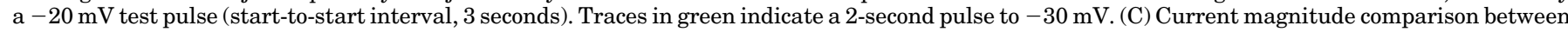

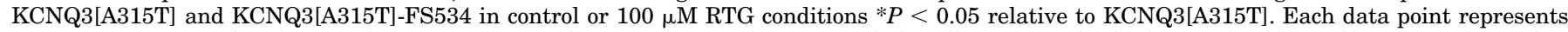

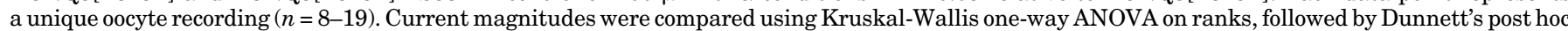
test to compare with the KCNQ3[A315T] control group. 
A

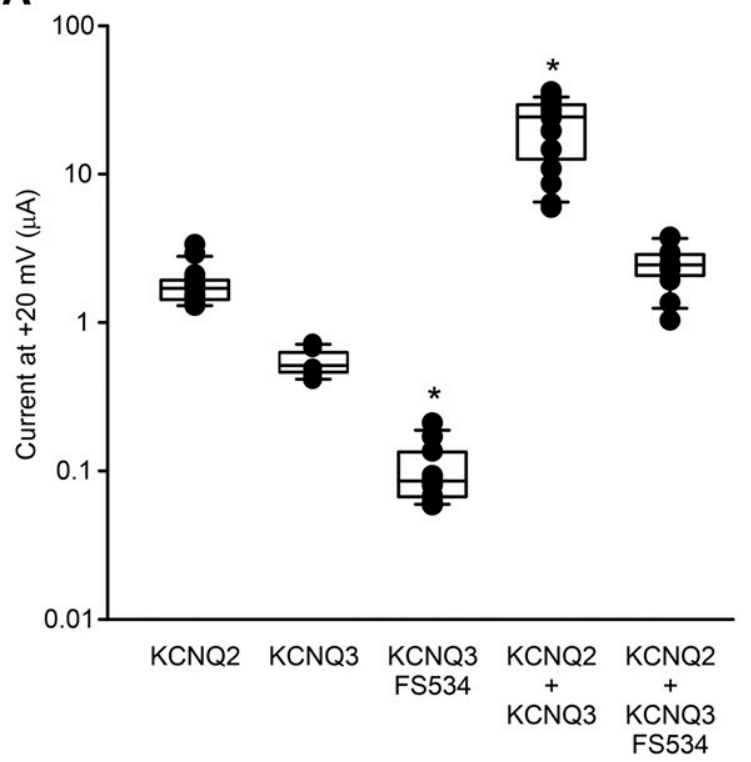

B

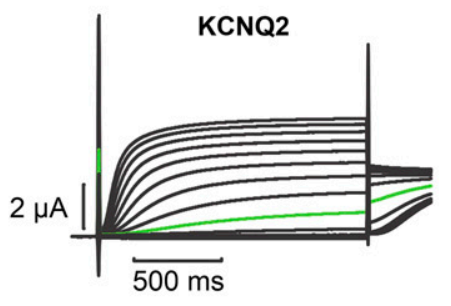

C

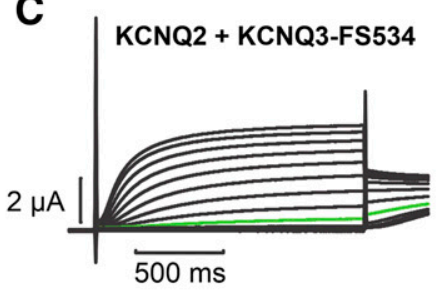

D

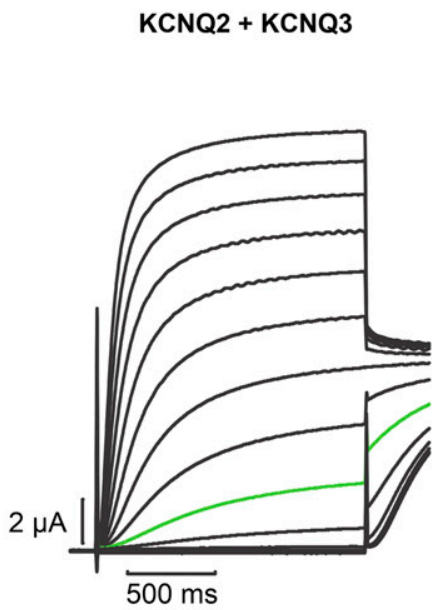

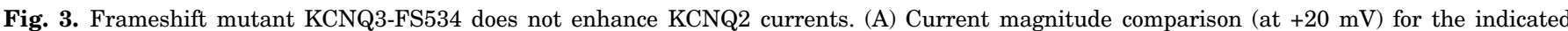

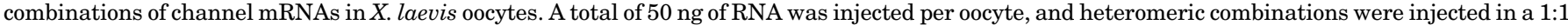

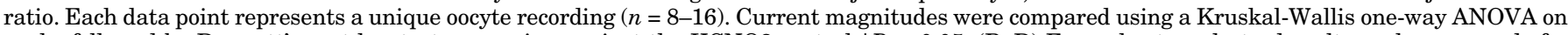

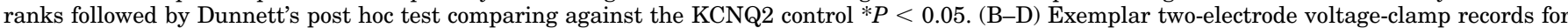

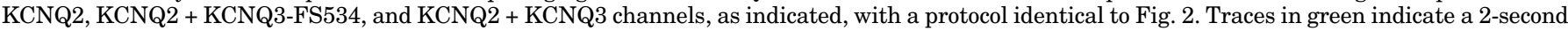
pulse to $-30 \mathrm{mV}$.

whether KCNQ3-FS534 is an "inert" contributor (fails to assemble and influence KCNQ2) or whether KCNQ3-FS534 assembles with KCNQ2 but fails to promote current expression of heteromeric channels.

Biophysical Assessment of Assembly of KCNQ3-FS534 with KCNQ2. Conductance-voltage relationships were collected to determine whether KCNQ3-FS534 subunits could influence channel gating or RTG sensitivity (Fig. 4). In the absence of RTG, KCNQ2 homomeric channels exhibited a $V_{1 / 2}$ of $-36.6 \pm 2.7 \mathrm{mV}$, whereas the KCNQ2 + KCNQ3-FS534 combination exhibited a shifted $\mathrm{V}_{1 / 2}$ of $-25.6 \pm 3.3 \mathrm{mV}$ (Fig. 4 , $\mathrm{A}$ and $\mathrm{C}$ ). In the presence of RTG, KCNQ2-5 channels exhibit a marked hyperpolarizing shift of voltage dependence (Tatulian et al., 2001; Tatulian and Brown, 2003; Kim et al., 2015). In our study, the RTG-mediated gating shift was $-41.2 \mathrm{mV}$ in KCNQ2 homomeric channels and was moderately smaller at $-36.2 \mathrm{mV}$ in KCNQ2 + KCNQ3-FS534 (Fig. 4D). WT KCNQ2 + WT KCNQ3 heteromers exhibited a RTG-mediated voltage shift of $-59.7 \mathrm{mV}$, with a $\mathrm{V}_{1 / 2}$ of $-37.5 \pm 4.6 \mathrm{mV}$ in control conditions (Fig. 4D). Conductancevoltage relationships for homomeric KCNQ2 and the wild-type heteromeric KCNQ2 + KCNQ3 combination are superimposed (gray curves in Fig. 4C) for comparison with KCNQ2 + KCNQ3FS534, illustrating modest differences in the $\mathrm{V}_{1 / 2}$ in control and the RTG-mediated shift in $\mathrm{V}_{1 / 2}$, when KCNQ2 is coexpressed with KCNQ3-FS534. Although small, these effects hinted that the KCNQ3-FS534 subunit may assemble with KCNQ2 and moderately influence its voltage dependence and RTG sensitivity.

Pharmacological Assessment of KCNQ2/3 Channel Assembly. In contrast to these effects, Lauritano et al. (2019) described an absence of any biophysical effects of KCNQ3FS534, using similar approaches but in a mammalian cell line. They also reported that the KCNQ3-FS534 mRNA was prone to nonsense-mediated mRNA decay, and this dramatically reduced expression may have also minimized the appearance of any biophysical effects of KCNQ3-FS534. We devised an alternative approach to test heteromeric assembly of KCNQ2 and KCNQ3. We recently reported how subunit composition alters sensitivity of channels to subtype-specific Kv7/KCNQ activator compounds (Wang et al., 2018a). We assessed these differences by exposing channels to $100 \mu \mathrm{M}$ ICA-069673 and delivering voltage step protocols as described in Figure 5. Channels were depolarized to $+20 \mathrm{mV}$ to fully activate channels and bind ICA-069673, which exhibits strict state dependence and only interacts with activated channels (Wang et al., 2018b). This was followed by a repolarizing step to a range of voltages ( -20 to $-120 \mathrm{mV}$ in $20 \mathrm{mV}$ steps) to allow drug unbinding and channel closure. Lastly, we stepped to $+20 \mathrm{mV}$ to assess the magnitude of instantaneous current (a reflection of the extent of deactivation that occurred during the repolarizing voltage step, highlighted with arrows in Fig. 5, A and B). This protocol distinguishes clearly between KCNQ2 homotetramers and KCNQ2/KCNQ3 heterotetramers, based on the magnitude of instantaneous current relative to the total current. We chose to quantify the magnitude of instantaneous current rather than the deactivation kinetics because extremely slow deactivation in the presence of ICA-069673 was difficult to quantify consistently. The voltage sensor-targeted drug ICA-069673 causes extremely slow deactivation of KCNQ2 homotetrameric channels (Fig. 5B) resulting in a very large instantaneous current (Fig. 5, B and C). These effects are markedly attenuated in heteromeric KCNQ2/KCNQ3 channels (Fig. 5, A and C). Illustrated over a range of hyperpolarization voltages (Fig. 5D), the weaker ICA-069673 sensitivity of KCNQ2/KCNQ3 heteromeric channels is apparent, resulting in a less prominent instantaneous current compared with KCNQ2 homomeric channels. This ability to close in the 
A

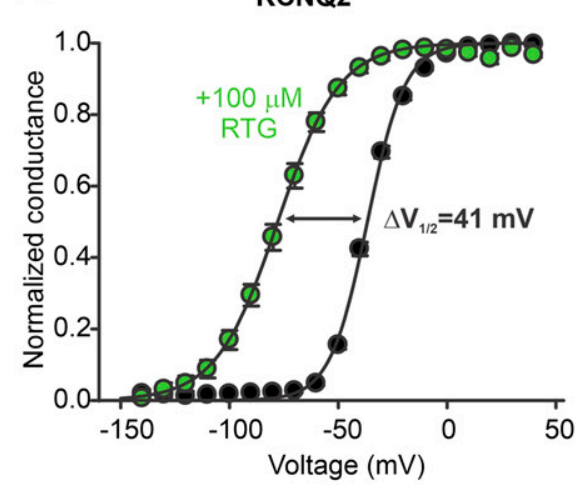

B

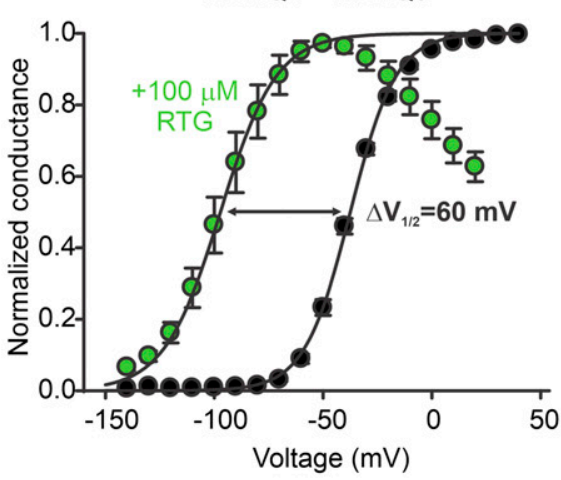

C

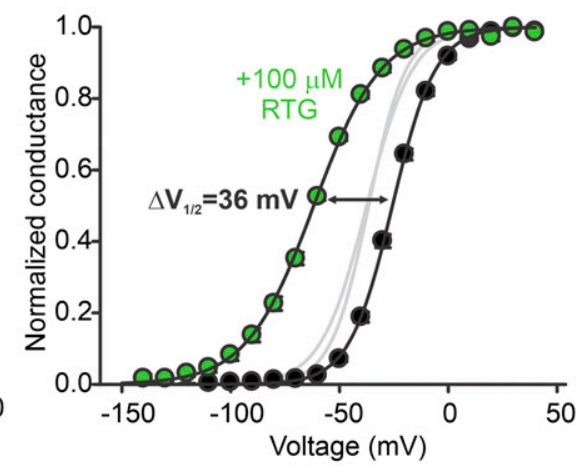

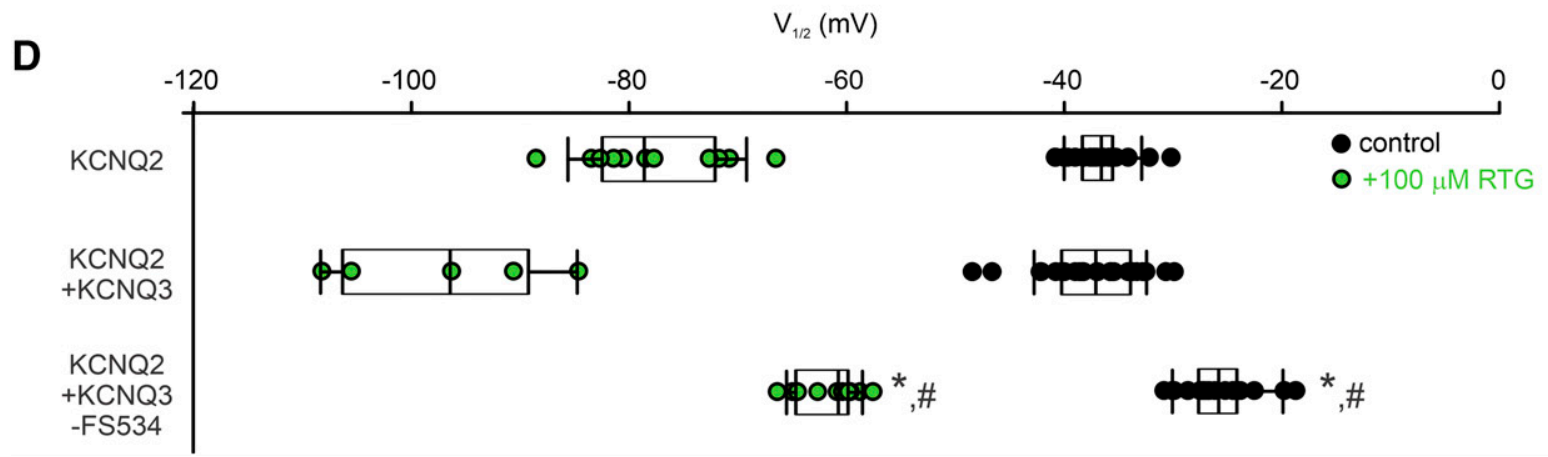

Fig. 4. Altered voltage dependence and RTG response of KCNQ2 coexpressed with KCNQ3-FS534. (A-C) Summary conductance-voltage relationships from KCNQ2 (A), KCNQ2 + KCNQ3 (B), and KCNQ2 + KCNQ3-FS534 (C) expressed in X. laevis oocytes. Gray curves in (C) represent conductancevoltage relationships from KCNQ2 and KCNQ2 + KCNQ3 in the absence of RTG for comparison with KCNQ2 + KCNQ3-FS534. (D) Cell-by-cell illustration of $\mathrm{V}_{1 / 2}$ for each channel combination in the presence or absence of $100 \mu \mathrm{M}$ RTG. Fitted gating parameters for KCNQ2 were, no RTG: $\mathrm{V}_{1 / 2}=-36.6 \pm 2.7 \mathrm{mV}, \mathrm{k}=8.6 \pm 1.1 ; 100 \mu \mathrm{M} \mathrm{RTG}: \mathrm{V}_{1 / 2}=-77.8 \pm 6.6 \mathrm{mV}, \mathrm{k}=14.1 \pm 4.3$. KCNQ2 + KCNQ3 fitted gating parameters were, no RTG: $\mathrm{V}_{1 / 2}=-37.5 \pm 4.6 \mathrm{mV}, \mathrm{k}=10.5 \pm 1.7 ; 100 \mu \mathrm{M}$ RTG: $\mathrm{V}_{1 / 2}=-97.2 \pm 9.9 \mathrm{mV}, \mathrm{k}=12.9 \pm 2.0 . \mathrm{KCNQ} 2+$ KCNQ3-FS534 fitted gating parameters were, no RTG: $\mathrm{V}_{1 / 2}=-25.6 \pm 3.3 \mathrm{mV}, \mathrm{k}=9.9 \pm 1.5 ; 100 \mu \mathrm{M}$ RTG: $\mathrm{V}_{1 / 2}=-61.8 \pm 2.8 \mathrm{mV}, \mathrm{k}=15.4 \pm 3.4$. Data in $(\mathrm{A})$ are means $\pm \mathrm{S} . \mathrm{E} . \mathrm{M}$. $\mathrm{V}_{1 / 2}$ compared between channel combinations in control and RTG conditions using a one-way ANOVA and the Holm-Sidak post hoc test. ${ }^{*} P<0.05$ relative to KCNQ2, ${ }^{\#} P<0.05$ relative to KCNQ2 + KCNQ3 heteromeric combination.

presence of high ICA-069673 concentrations is a signature of heteromeric KCNQ2/KCN3 channels and clearly distinguishes them from homomeric KCNQ2 channels.

Assembly of C-Terminally Truncated KCNQ3 with KCNQ2. We used ICA-069673 as a pharmacological tool to investigate assembly of KCNQ3-FS534 with KCNQ2 and extended this approach to a variety of KCNQ3 C-terminal truncations (Fig. 6). Our approach may be valuable for future studies of assembly or effects of channel mutants, as it can clearly demonstrate whether a KCNQ3 mutant assembles with KCNQ2 and generates functional channels (leading to altered pharmacological properties). In cases where channels retain full sensitivity to ICA-069673, this experiment does not directly distinguish between the possibility of dominantnegative effects of a KCNQ3 mutant versus failure of the mutant to assemble with KCNQ2 (in both cases the currents would be generated predominantly by KCNQ2 homomers, leading to full sensitivity to ICA-069673). However, the demonstration of altered ICA-069673 sensitivity relative to KCNQ2 homomers provides strong evidence that a KCNQ3 mutant effectively assembles with KCNQ2 to form functional channels.

Using this approach we observed that prominent C-terminal truncations of KCNQ3 are well tolerated in terms of assembly with KCNQ2 (Fig. 6). Instantaneous currents (after repolarization to $-100 \mathrm{mV}$ ) are illustrated in sample sweeps (Fig. 6A), along with mean data for instantaneous current magnitude across a range of repolarization voltages (Fig. 6, B and C). The $\Delta$ C301 truncation was not distinguishable from WT KCNQ3 in this assay. Truncations as large as 340 amino acids, resulting in deletion of nearly the entire $\mathrm{C}$ terminus up to the $\mathrm{A}$ and $\mathrm{B}$ helices (required for calmodulin binding), still supported prominent assembly with KCNQ2, as indicated by decreased responsiveness to ICA-069673 (Fig. 6, $\mathrm{B}$ and C). However, both KCNQ3-FS534 and KCNQ3- $\Delta$ C340 exhibited intermediate responses to ICA-069673, suggesting that the region between $\Delta \mathrm{C} 340$ and $\Delta \mathrm{C} 301$ is an important segment where effects on heteromeric KCNQ2/ KCNQ3 assembly start to become apparent. Since helix A and helix B appear to function as a unit and assemble with calmodulin (shown in green in Fig. 6A), we did not test progressive deletions of this region, but we found that after deletion of the bulk of the $\mathrm{C}$ terminus, including helices $\mathrm{A}$ and B, KCNQ3 finally appeared to be "inert" and failed to influence ICA-069673 sensitivity when coexpressed with KCNQ2 (Fig. 6, B and C).

Titration of KCNQ2/KCNQ3 Ratios. An important uncertainty in a heterologous system is whether overexpression of proteins drives the assembly of a complex, overcoming the loss of structural elements that might be involved in specific assembly. To investigate this possibility, we injected oocytes with smaller amounts of KCNQ3 (WT or $\Delta$ C301) relative to 
A
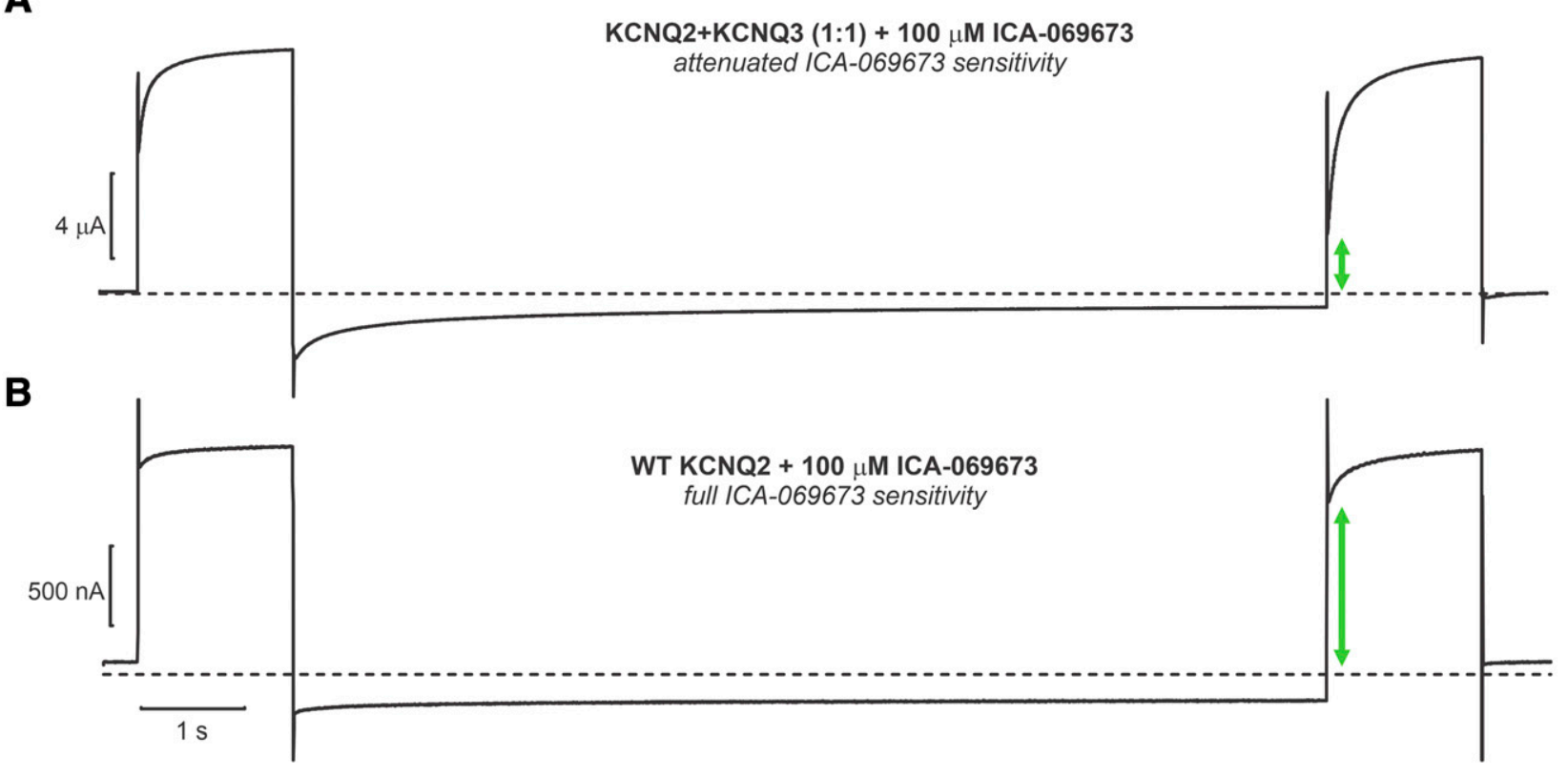
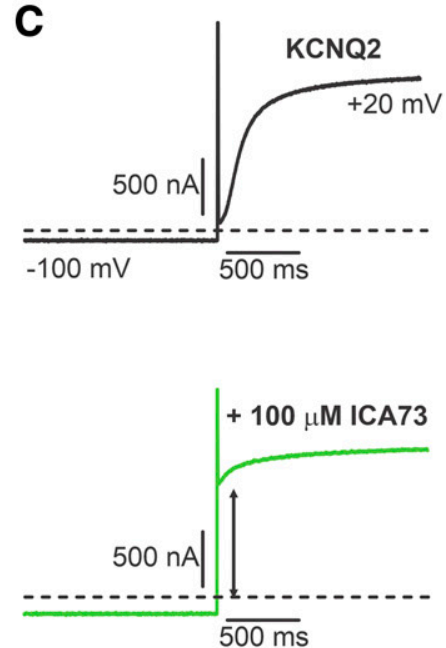
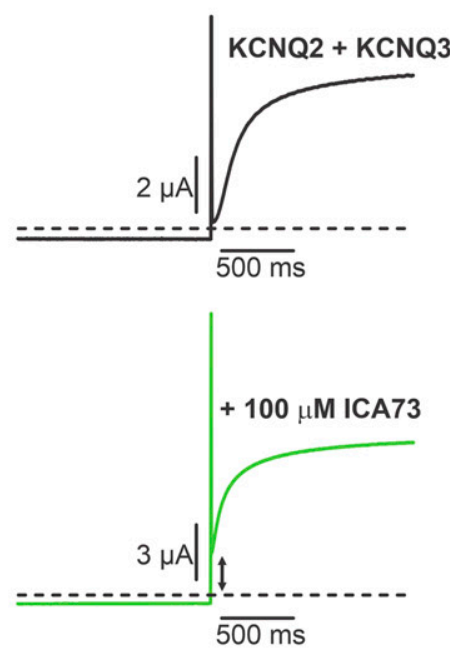

D

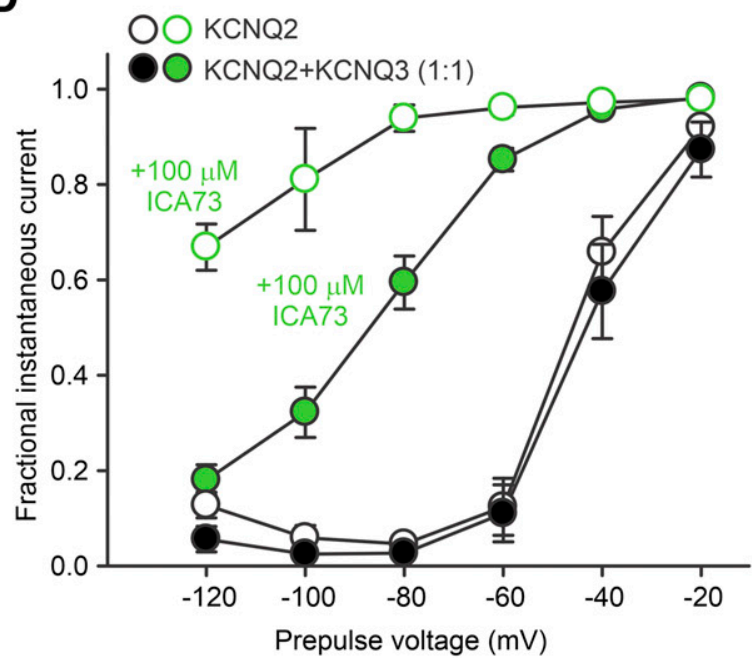

Fig. 5. Attenuated effects of ICA-069673 on heteromeric KCNQ2 + KCNQ3 channels. (A and B) Exemplar X. laevis oocyte two-electrode voltage-clamp records for measurement of instantaneous current. Injected oocytes were held at a prepulse voltage of $-100 \mathrm{mV}$ for 10 seconds, then pulsed to $+20 \mathrm{mV}$ for 2 seconds. Green arrows highlight measurement of instantaneous current fraction. (C) Exemplar traces illustrating channel activation after - $100 \mathrm{mV}$ prepulses in the absence and presence of ICA-069673 (5.9\% $\pm 0.9 \%$ and $81.1 \% \pm 3.8 \%$, respectively, for $\mathrm{KCNQ} 2,2.5 \% \pm 0.3 \%$ and $32.3 \% \pm 2.0 \%$, respectively, for KCNQ2 + KCNQ3). (D) Fractional instantaneous current was measured after a range of prepulse voltages $(n=7-12)$. Data in (D) are presented as means \pm S.E.M.

KCNQ2 and investigated current magnitude and ICA-069673 sensitivity (using the instantaneous current assay described in Figs. 5 and 6). Coexpression of KCNQ2 with KCNQ3 caused prominent current enhancement (Fig. 7B), together with substantial suppression of ICA-069673 sensitivity (Fig. 7A), with even the smallest ratio tested (4:1 ratio of $20 \mathrm{ng} \mathrm{KCNQ} 2$ and $5 \mathrm{ng}$ KCNQ3) (Fig. 7). Coinjection with the same 4:1 ratio of KCNQ2 and KCNQ3- $\Delta$ C301 did not potentiate heteromeric channel current to the same extent as WT KCNQ3 (Fig. 7B). However, KCNQ3- $\Delta$ C301 subunits exhibited similar loss of ICA-069673 sensitivity as WT KCNQ3, suggesting a strong propensity to assemble with KCNQ2 (Fig. 7A). Consistent with our truncation scan in Figure 6, assembly was clearly attenuated for the KCNQ3- $\Delta$ C340 mutant, along with current potentiation when coexpressed with KCNQ2 (Fig. 7).

Figure 8 summarizes the effects of $1: 1$ coinjection of various KCNQ3 truncations with KCNQ2, highlighting patterns related to assembly versus expression of these different subunit combinations. A general pattern emerges that progressive truncation of KCNQ3 causes a gradual reduction of total current expression (Fig. 8B). Modest truncations of KCNQ3 (e.g., $\triangle \mathrm{C} 42$ ) support the potentiation of current after coassembly with KCNQ2, but this effect is largely lost with truncations of $\Delta$ C301 or greater. There is a slight mismatch between the effect on current and the effect on pharmacologically assessed heteromeric assembly, in which KCNQ2 assembly with KCNQ3- $\Delta$ C301 
A

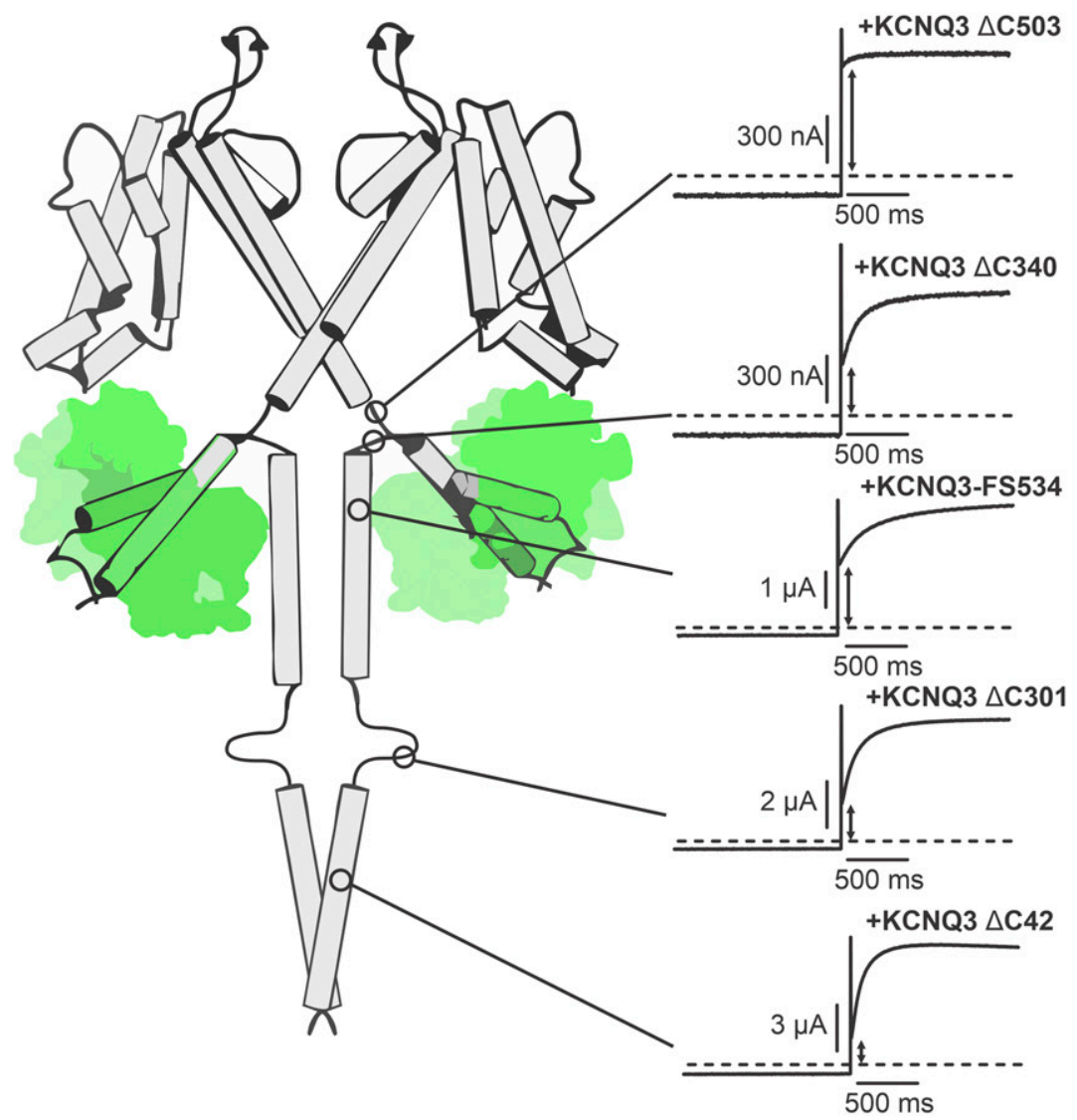

B

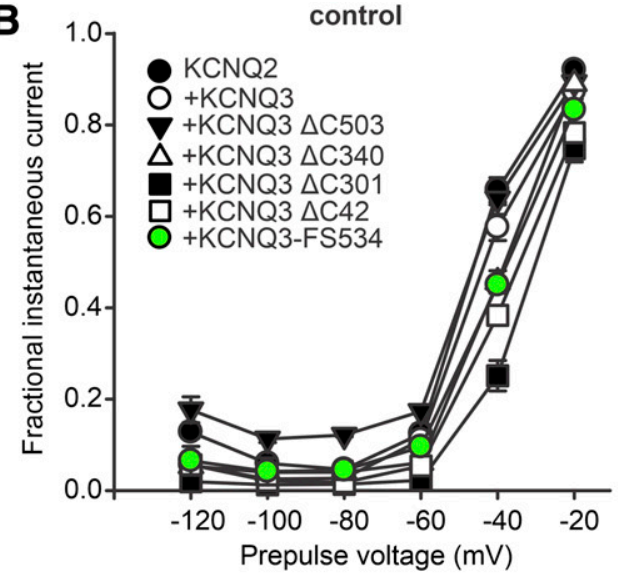

C

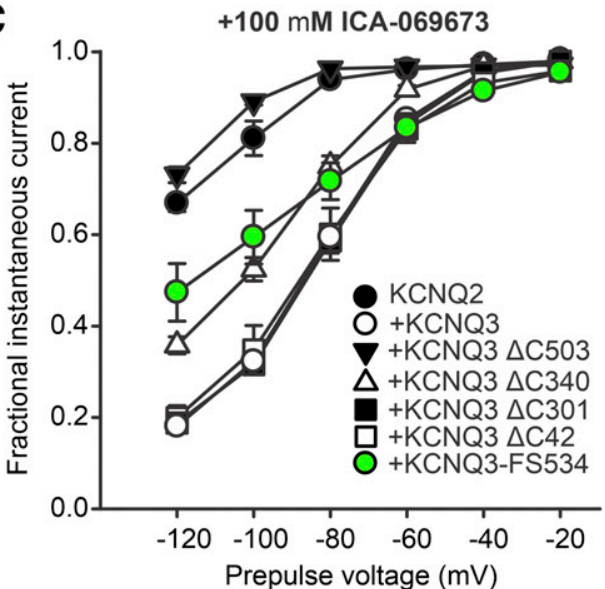

Fig. 6. Functional assessment of assembly of KCNQ3 truncations with KCNQ2. (A) KCNQ structural model illustrating the location of KCNQ truncations tested in the study. Exemplar two-electrode voltage-clamp records illustrating instantaneous current for KCNQ2 + KCNQ3 truncations with respect to the positions of truncations. Calmodulin is illustrated in green. Coinjections were controlled for total mRNA at 50 ng, with a 1:1 KCNQ2: KCNQ3 ratio. (B and C) Instantaneous currents were measured after a range of prepulse voltages, using the same protocol as Fig. 5, A and B. Instantaneous currents were measured in control (B) or $100 \mu \mathrm{M} \mathrm{ICA}-069673$ (C) $(n=4-12$ for control, $n=7-11$ for drug condition). Data in (B and C) are presented as means \pm S.E.M.

appears to be equally favorable as with WT KCNQ3, although it cannot potentiate currents to the same degree.

\section{Discussion}

Structural elements that drive assembly of KCNQ channels have been previously investigated and have primarily focused on the long $\mathrm{C}$ termini of this channel family (Howard et al., 2007; Haitin and Attali, 2008; Sun and MacKinnon, 2017). Our study investigated a series of C-terminal truncations of KCNQ3, using a subtype-selective KCNQ2 activator compound (ICA-069673) to distinguish heteromeric KCNQ2/ KCNQ3 channels from homomeric KCNQ2. This approach clearly reveals that large C-terminal truncations of KCNQ3, including a disease-linked frameshift mutation, can assemble efficiently with WT KCNQ2.

Although much attention has been devoted to the role of the $\mathrm{N}$-terminal $\mathrm{T} 1$ domain in regulating function and assembly of the Kv1-4 gene family (along with the silent Kv5,6,8,9 subfamilies), the Kv7/KCNQ gene family lacks an N-terminal $\mathrm{T} 1$ domain and must have other determinants that control assembly (Li et al., 1992; Kreusch et al., 1998; Bixby et al., 1999; Schwake et al., 2003, 2006). In terms of determining stoichiometry of different subunits, or specificity of assembly, there are many unanswered questions. Native M-channels are usually ascribed to contain KCNQ2 and KCNQ3, and it has been suggested that these channels comprise a 1:1 stoichiometry as determined by tetraethylammonium (TEA) sensitivity (Hadley et al., 2000, 2003). However, it is not known how this assembly is determined or whether other stoichiometries are excluded (Bal et al., 2008; Stewart et al., 2012). Similarly, it is generally accepted that KCNQ2 and KCNQ5 subunits do not assemble to form functional channels, but it is not known how this exclusion is controlled (Schroeder et al., 2000; Tzingounis et al., 2010). Lastly, KCNQ1 is excluded from assembly with "noncardiac" KCNQ subtypes KCNQ2-5, but this can be manipulated by swapping C-terminal sequences between KCNQ subtypes (Schwake et al., 2003).

We were motivated to investigate $\mathrm{C}$-terminal regulation of assembly of M-channels based on the identification of a C-terminal KCNQ3 truncation in a patient diagnosed with autism and general neurodevelopmental delay but lacking presentation of seizures. From a clinical perspective, we found this patient to be interesting, as KCNQ3 loss-of-function mutations are typically observed in children with neonatal epilepsy, but this hallmark symptom was absent in our proband. In addition, a recent report highlighted an identical frameshift in an Italian family with consanguineous marriage, in which 
A

Fractional instantaneous current, $-100 \mathrm{mV}$ prepulse

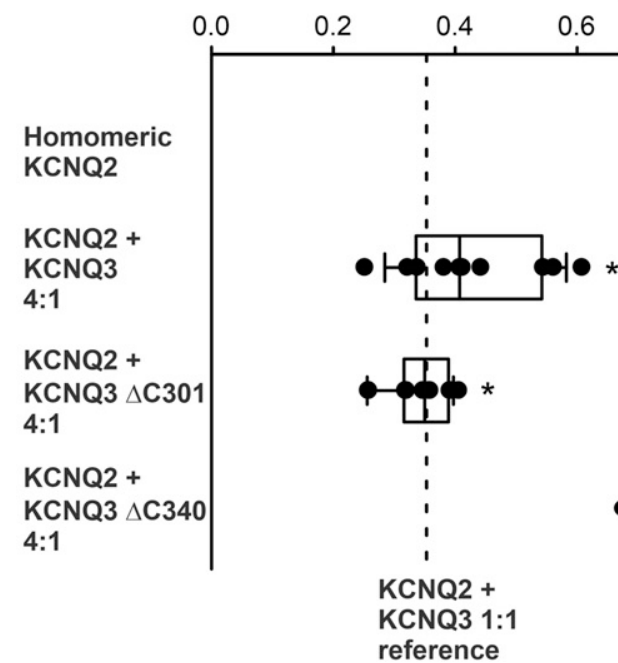

B

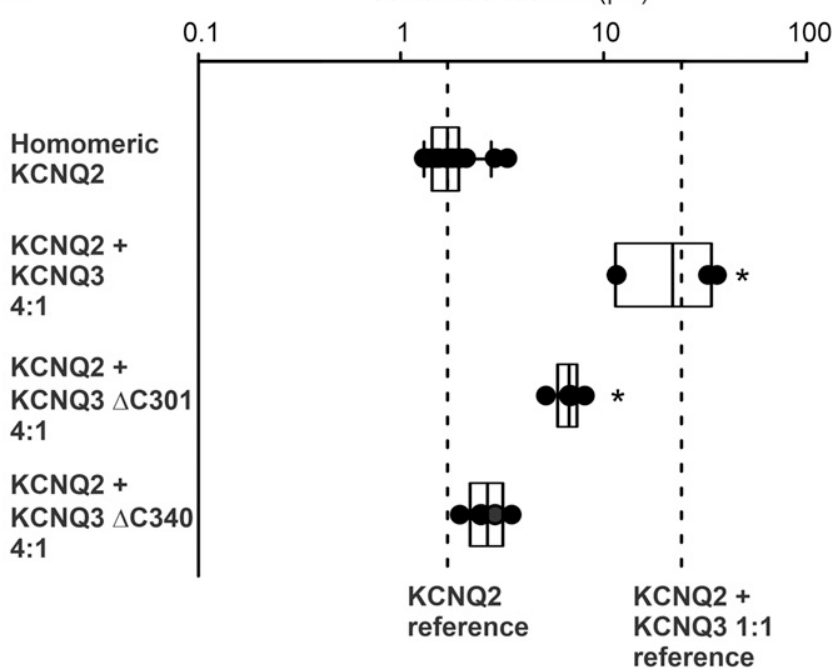

Fig. 7. KCNQ3 $\Delta$ C301 and WT KCNQ3 have a similar propensity to assemble with KCNQ2. (A) Fractional instantaneous currents in $100 \mu M$ ICA-069673 for indicated channel combinations ( $25 \mathrm{ng}$ total RNA injection) using the experimental protocol described in Fig. 5A. Dotted reference lines indicate the mean fractional instantaneous currents of homomeric KCNQ2 (mean $=81.1 \% \pm 3.8 \%, n=8$ ) and heteromeric KCNQ2 $+\mathrm{KCNQ} 31: 1$ mixture $($ mean $=32.3 \% \pm 2.0 \%, n=7)$. Each data point represents a unique oocyte recording $(n=4-10)$. (B) Current amplitudes of the same oocytes when pulsed to $+20 \mathrm{mV}$. Dotted reference lines indicate the mean current amplitude of homomeric KCNQ2 and heteromeric KCNQ2 + KCNQ3 1:1 mixture. Each data point represents a unique oocyte recording $(n=4-16)$. Data were compared using a Kruskal-Wallis ANOVA on ranks, followed by Dunnett's post hoc test comparing against the homomeric KCNQ2 control. ${ }^{*} P<0.05$.

homozygous patients exhibited much more prominent penetrance of neurologic features (Lauritano et al., 2019). Although there was some uncertainty involving diagnosis and symptoms of a likely heterozygous relative in the Italian study, both heterozygous parents were only modestly affected by this large truncation of the $\mathrm{C}$ terminus. In contrast, the proband identified
A

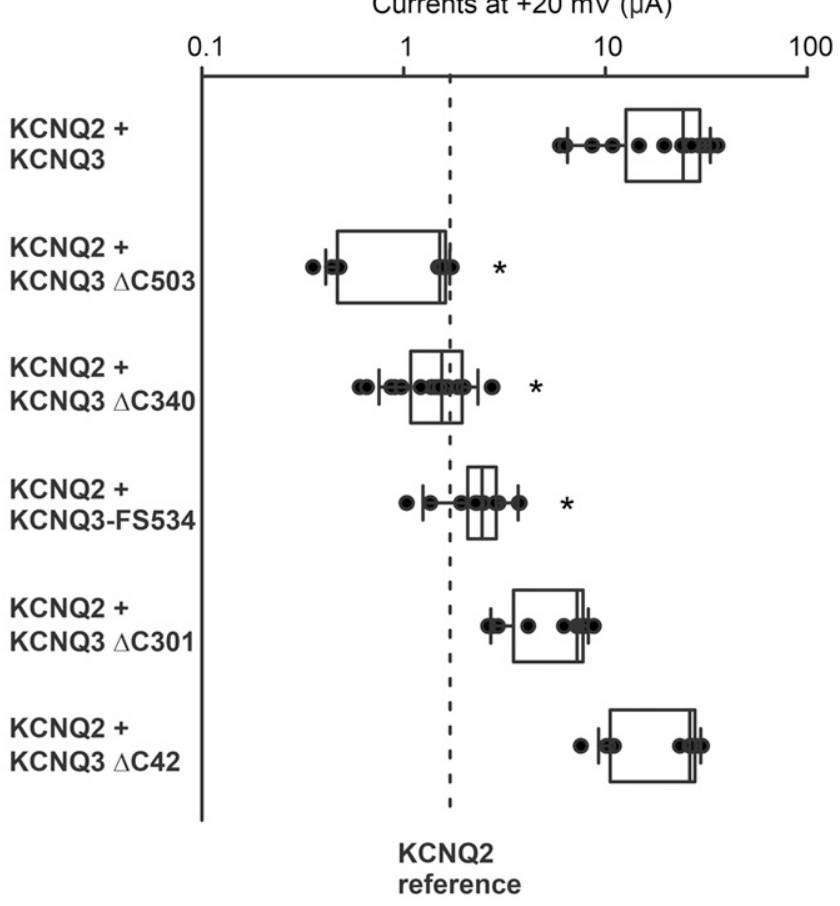

B

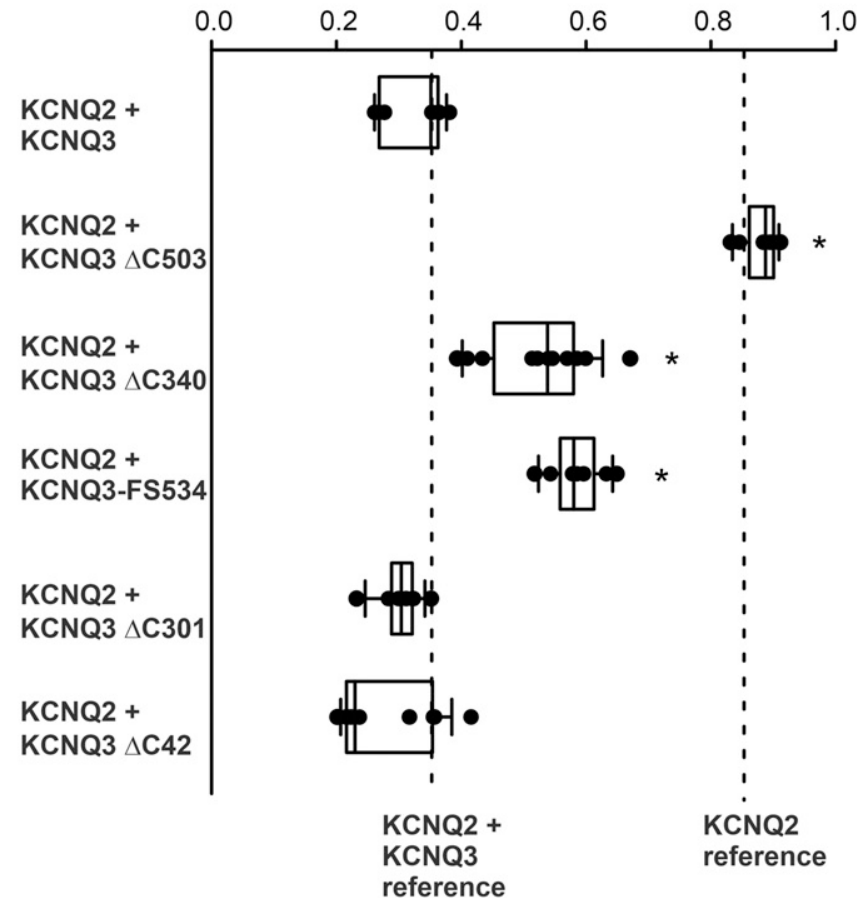

Fig. 8. Most KCNQ3 truncations cannot enhance KCNQ2 current to wild-type levels. (A) Current amplitudes of various 1:1 KCNQ2-KCNQ3 coinjections in $X$. laevis oocytes when pulsed to $+20 \mathrm{mV}$. Although KCNQ3- $\Delta$ C503 is the only construct unable to assemble with KCNQ2, having the ability to assemble is not sufficient to return current levels back to normal. Each data point represents a unique oocyte recording $(n=12-20$ for coinjections). The dotted line reflects the average current amplitude of KCNQ2 homomers (mean $=1.81 \pm 0.14 \mu \mathrm{A}, n=16$ ) in X. laevis oocytes. (B) Fractional instantaneous current in $100 \mu \mathrm{M}$ ICA-069673 for indicated 1:1 coinjections of KCNQ2 + KCNQ3 (full length or truncated). Dotted reference lines indicate the mean fractional instantaneous currents of homomeric KCNQ2 and heteromeric KCNQ2 + KCNQ3 1:1 mixture. Data were compared using a Kruskal-Wallis ANOVA on ranks, followed by Dunnett's post hoc test comparing against the KCNQ2 + KCNQ3 control. ${ }^{*} P<0.05$. 
in our study exhibited notable autistic traits and neurodevelopmental disability, including challenges with social reciprocity, receptive and expressive language, fine motor skills, and markedly reduced sustained attention. These findings suggest that differences in their genetic background may have affected the penetrance of the defect emerging from the KCNQ3-FS534 mutation. The noted mild deficits in the proband's relatives suggest additional unknown genetic contributors, although whole exome sequencing did not reveal any causative mutations in any genes (in any family members) linked to the reported phenotype.

Consistent with prior findings, the major defect attributed to the KCNQ3-FS534 mutation is disrupted expression of functional KCNQ2/KCNQ3 heteromeric M-channels. Lauritano et al. (2019) provide compelling evidence that the KCNQ3-FS534 mutation is poorly expressed in heterologous cell lines and patient-derived fibroblasts, attributed to nonsense-mediated decay of mRNA (Lauritano et al., 2019). In addition, upon coexpression with KCNQ2, they observed little evidence for the generation of functional heteromeric channels. In our experiments using Xenopus oocytes rather than cell lines, we observed substantial assembly of KCNQ2 with a wide range of truncated forms of KCNQ3. It is important to note that since we are injecting mRNA, no mRNA splicing occurs during gene expression, and so nonsense-mediated mRNA decay may not have a strong influence on expression in the Xenopus oocyte system used for our experiments. Thus, our findings are likely most relevant in the context of understanding determinants of heteromeric KCNQ channel expression, rather than cell biologic processes that might also influence mRNA/protein abundance.

An interesting aspect of our study is the demonstration of an alternative approach to identify heteromerization of KCNQ2 with other KCNQ subtypes (especially KCNQ3, and potentially KCNQ5) based on sensitivity to the voltage sensor-targeted drug ICA-069673. We previously demonstrated that ICA-069673 effects depend strongly on subunit composition of M-channels. KCNQ2 homomeric channels exhibit extreme deceleration of deactivation, requiring $\sim 10-20$-second hyperpolarizing steps to allow the drug to unbind from multiple subunits and permit channel closure (Wang et al., 2018a). In contrast, heteromeric channels comprising KCNQ2 mixed with ICA-069673-insensitive subunits such as KCNQ3 exhibit faster channel closure upon hyperpolarization (Wang et al., 2017, 2018a,b). The mechanism of coupling between voltage sensors and the KCNQ channel pore that underlies this functional outcome is not yet clear, but this effect is useful as a diagnostic tool for heteromeric assembly of KCNQ2 with other subtypes. It has previously been common to exploit the differential TEA sensitivity of KCNQ2 and KCNQ3 to demonstrate subunit heteromerization (Hadley et al., 2000, 2003; Soldovieri et al., 2016). Our findings provide an alternative approach to this experimental question and may benefit by using a more specific pharmacological tool.

The demonstration of efficient assembly of large C-terminal KCNQ3 truncations with KCNQ2 was unexpected, as prior studies of KCNQ channel assembly have attributed an important role for the $\mathrm{C}$ terminus in promoting channel assembly (Maljevic et al., 2003; Schwake et al., 2003, 2006; Haitin and Attali, 2008). It is noteworthy that many prior studies on this topic used chimeric strategies of replacing $\mathrm{C}$-terminal segments of KCNQ1 with KCNQ2 or KCNQ3 and used current potentiation as an indicator of whether various chimeric subunits could assemble with compatible M-channel subunits (Maljevic et al., 2003; Schwake et al., 2003, 2006). Although these studies clearly established important regulatory elements in the $\mathrm{C}$ terminus, it was not demonstrated whether effects on current expression arose from altered assembly versus an effect on maturation or proteostasis. Later studies provided some evidence that prominent C-terminal deletions of KCNQ2 or KCNQ3 retain some propensity to assemble, based on pharmacological assessment of TEA sensitivity (Nakajo and Kubo, 2008). Structural studies indicate that the $C$ termini of Kv7/KCNQ channels can self-assemble into coiled-coil structures, with a variety of proposed functions, including promotion of tetrameric channel assembly, and possibly acting as a scaffold for association with regulatory proteins (Howard et al., 2007; Sun and MacKinnon, 2017). Our findings indicate that regulators of assembly versus proteostasis/maturation are likely controlled by distinct channel segments and that pharmacological assays may be a useful tool to investigate subunit composition in future studies.

In summary, our study introduces a new and useful approach to investigate heteromerization of KCNQ channels based on subtype-specific pharmacological tools. Our findings illustrate that long truncations of the KCNQ3 $\mathrm{C}$ terminus are tolerated in terms of assembly with KCNQ2 but fail to promote functional current expression (unlike full-length KCNQ3).

\section{Authorship Contributions}

Participated in research design: Li, Marco, Kurata.

Conducted experiments: Li, Maghera, Lamothe, Marco.

Performed data analysis: Li, Marco, Kurata.

Wrote or contributed to the writing of the manuscript: Li, Maghera, Lamothe, Marco, Kurata.

\section{References}

Adams PR and Brown DA (1982) Synaptic inhibition of the M-current: slow excitatory post-synaptic potential mechanism in bullfrog sympathetic neurones. J Physiol 332:263-272.

Bal M, Zhang J, Zaika O, Hernandez CC, and Shapiro MS (2008) Homomeric and heteromeric assembly of KCNQ (Kv7) K+ channels assayed by total internal reflection fluorescence/fluorescence resonance energy transfer and patch clamp analysis. J Biol Chem 283:30668-30676.

Biervert C, Schroeder BC, Kubisch C, Berkovic SF, Propping P, Jentsch TJ, and Steinlein OK (1998) A potassium channel mutation in neonatal human epilepsy. Science 279:403-406.

Bixby KA, Nanao MH, Shen NV, Kreusch A, Bellamy H, Pfaffinger PJ, and Choe S (1999) Zn2+-binding and molecular determinants of tetramerization in voltagegated K+ channels. Nat Struct Biol 6:38-43.

Brown DA and Adams PR (1980) Muscarinic suppression of a novel voltage-sensitive $\mathrm{K}+$ current in a vertebrate neurone. Nature 283:673-676.

Charlier C, Singh NA, Ryan SG, Lewis TB, Reus BE, Leach RJ, and Leppert M (1998) A pore mutation in a novel KQT-like potassium channel gene in an idiopathic epilepsy family. Nat Genet 18:53-55.

Cooper EC, Aldape KD, Abosch A, Barbaro NM, Berger MS, Peacock WS, Jan YN, and Jan LY (2000) Colocalization and coassembly of two human brain M-type potassium channel subunits that are mutated in epilepsy. Proc Natl Acad Sci USA 97:4914-4919.

Etxeberria A, Santana-Castro I, Regalado MP, Aivar P, and Villarroel A (2004) Three mechanisms underlie KCNQ2/3 heteromeric potassium M-channel potentiation. $J$ Neurosci 24:9146-9152.

Gómez-Posada JC, Etxeberría A, Roura-Ferrer M, Areso P, Masin M, Murrell-Lagnado RD, and Villarroel A (2010) A pore residue of the KCNQ3 potassium M-channel subunit controls surface expression. J Neurosci 30:9316-9323.

Hadley JK, Noda M, Selyanko AA, Wood IC, Abogadie FC, and Brown DA (2000) Differential tetraethylammonium sensitivity of KCNQ1-4 potassium channels. $\mathrm{Br} J$ Pharmacol 129:413-415.

Hadley JK, Passmore GM, Tatulian L, Al-Qatari M, Ye F, Wickenden AD, and Brown DA (2003) Stoichiometry of expressed KCNQ2/KCNQ3 potassium channels and subunit composition of native ganglionic $\mathrm{M}$ channels deduced from block by tetraethylammonium. J Neurosci 23:5012-5019.

Haitin Y and Attali B (2008) The C-terminus of Kv7 channels: a multifunctional module. J Physiol 586:1803-1810.

Hill AS, Nishino A, Nakajo K, Zhang G, Fineman JR, Selzer ME, Okamura Y, and Cooper EC (2008) Ion channel clustering at the axon initial segment and node of Ranvier evolved sequentially in early chordates. PLoS Genet 4:e1000317. 
Howard RJ, Clark KA, Holton JM, and Minor DL Jr. (2007) Structural insight into KCNQ (Kv7) channel assembly and channelopathy. Neuron 53:663-675.

Kim RY, Yau MC, Galpin JD, Seebohm G, Ahern CA, Pless SA, and Kurata HT (2015) Atomic basis for therapeutic activation of neuronal potassium channels. Nat Commun 6:8116.

Kreusch A, Pfaffinger PJ, Stevens CF, and Choe S (1998) Crystal structure of the tetramerization domain of the Shaker potassium channel. Nature 392:945-948.

Lauritano A, Moutton S, Longobardi E, Tran Mau-Them F, Laudati G, Nappi P, Soldovieri MV, Ambrosino P, Cataldi M, Jouan T, et al. (2019) A novel homozygous KCNQ3 loss-of-function variant causes non-syndromic intellectual disability and neonatal-onset pharmacodependent epilepsy. Epilepsia Open 4:464-475.

Lerche H, Shah M, Beck H, Noebels J, Johnston D, and Vincent A (2013) Ion channels in genetic and acquired forms of epilepsy. $J$ Physiol 591:753-764.

Li M, Jan YN, and Jan LY (1992) Specification of subunit assembly by the hydrophilic amino-terminal domain of the Shaker potassium channel. Science 257:1225-1230.

Maljevic S, Lerche C, Seebohm G, Alekov AK, Busch AE, and Lerche H (2003) C-terminal interaction of KCNQ2 and KCNQ3 K+ channels. J Physiol 548: 353-360.

Maljevic S and Lerche H (2014) Potassium channel genes and benign familial neonatal epilepsy. Prog Brain Res 213:17-53.

Miceli F, Soldovieri MV, Joshi N, Weckhuysen S, Cooper E, and Taglialatela M (1993a) KCNQ2-related disorders, in GeneReviews ${ }^{\circledR}$ (Adam MP, Ardinger HH, Pagon RA, Wallace SE, Bean LJ, Stephens K, and Amemiya A, University of Washington, Seattle, Seattle.

Miceli F, Soldovieri MV, Joshi N, Weekhuysen S, Cooper EC, and Tagliatela M (1993b) KCNQ3-related disorders, in GeneReviews ${ }^{\circledR}$ (Adam MP, Ardinger HH, Pagon RA, Wallace SE, Bean LJ, Stephens K, and Amemiya A, University of Washington, Seattle.

Millichap JJ, Park KL, Tsuchida T, Ben-Zeev B, Carmant L, Flamini R, Joshi N, Levisohn PM, Marsh E, Nangia S, et al. (2016) KCNQ2 encephalopathy: features, mutational hot spots, and ezogabine treatment of 11 patients. Neurol Genet 2:e96.

Nakajo K and Kubo Y (2008) Second coiled-coil domain of KCNQ channel controls current expression and subfamily specific heteromultimerization by salt bridge networks. J Physiol 586:2827-2840.

Padilla K, Wickenden AD, Gerlach AC, and McCormack K (2009) The KCNQ2/3 selective channel opener ICA-27243 binds to a novel voltage-sensor domain site. Neurosci Lett 465:138-142.

Schmitt N, Schwarz M, Peretz A, Abitbol I, Attali B, and Pongs O (2000) A recessive C-terminal Jervell and Lange-Nielsen mutation of the KCNQ1 channel impairs subunit assembly. EMBO J 19:332-340.

Schroeder BC, Hechenberger M, Weinreich F, Kubisch C, and Jentsch TJ (2000) KCNQ5, a novel potassium channel broadly expressed in brain, mediates M-type currents. J Biol Chem 275:24089-24095.

Schwake M, Athanasiadu D, Beimgraben C, Blanz J, Beck C, Jentsch TJ, Saftig P, and Friedrich T (2006) Structural determinants of M-type KCNQ (Kv7) K+ channel assembly. J Neurosci 26:3757-3766.

Schwake M, Jentsch TJ, and Friedrich T (2003) A carboxy-terminal domain determines the subunit specificity of KCNQ K+ channel assembly. EMBO Rep 4:76-81.

Shapiro MS, Roche JP, Kaftan EJ, Cruzblanca H, Mackie K, and Hille B (2000) Reconstitution of muscarinic modulation of the KCNQ2/KCNQ3 K(+) channels that underlie the neuronal M current. J Neurosci 20:1710-1721.
Singh NA, Charlier C, Stauffer D, DuPont BR, Leach RJ, Melis R, Ronen GM, Bjerre I, Quattlebaum T, Murphy JV, et al. (1998) A novel potassium channel gene, KCNQ2, is mutated in an inherited epilepsy of newborns. Nat Genet 18:25-29.

Soldovieri MV, Ambrosino P, Mosca I, De Maria M, Moretto E, Miceli F, Alaimo A, Iraci N, Manocchio L, Medoro A, et al. (2016) Early-onset epileptic encephalopathy caused by a reduced sensitivity of Kv7.2 potassium channels to phosphatidylinositol 4,5-bisphosphate. Sci Rep 6:38167.

Stewart AP, Gómez-Posada JC, McGeorge J, Rouhani MJ, Villarroel A, MurrellLagnado RD, and Edwardson JM (2012) The Kv7.2/Kv7.3 heterotetramer assembles with a random subunit arrangement. J Biol Chem 287:11870-11877.

Suh B-C, Inoue T, Meyer T, and Hille B (2006) Rapid chemically induced changes of PtdIns(4,5)P2 gate KCNQ ion channels. Science 314:1454-1457.

Sun J and MacKinnon R (2017) Cryo-EM structure of a KCNQ1/CaM complex reveals insights into congenital long QT syndrome. Cell 169:1042-1050.e9.

Tatulian L and Brown DA (2003) Effect of the KCNQ potassium channel opener retigabine on single KCNQ2/3 channels expressed in CHO cells. J Physiol 549: $57-63$

Tatulian L, Delmas P, Abogadie FC, and Brown DA (2001) Activation of expressed KCNQ potassium currents and native neuronal M-type potassium currents by the anti-convulsant drug retigabine. J Neurosci 21:5535-5545.

Tzingounis AV, Heidenreich M, Kharkovets T, Spitzmaul G, Jensen HS, Nicoll RA, and Jentsch TJ (2010) The KCNQ5 potassium channel mediates a component of the afterhyperpolarization current in mouse hippocampus. Proc Natl Acad Sci USA 107:10232-10237.

Wang AW, Yang R, and Kurata HT (2017) Sequence determinants of subtype-specific actions of KCNQ channel openers. J Physiol 595:663-676.

Wang AW, Yau MC, Wang CK, Sharmin N, Yang RY, Pless SA, and Kurata HT (2018a) Four drug-sensitive subunits are required for maximal effect of a voltage sensor-targeted KCNQ opener. J Gen Physiol 150:1432-1443.

Wang CK, Lamothe SM, Wang AW, Yang RY, and Kurata HT (2018b) Pore- and voltage sensor-targeted KCNQ openers have distinct state-dependent actions. $J$ Gen Physiol 150:1722-1734.

Wang HS, Pan Z, Shi W, Brown BS, Wymore RS, Cohen IS, Dixon JE, and McKinnon D (1998) KCNQ2 and KCNQ3 potassium channel subunits: molecular correlates of the M-channel. Science 282:1890-1893.

Waterhouse A, Bertoni M, Bienert S, Studer G, Tauriello G, Gumienny R, Heer FT, de Beer TAP, Rempfer C, Bordoli L, et al. (2018) SWISS-MODEL: homology modelling of protein structures and complexes. Nucleic Acids Res 46 (W1):W296-W303.

Weckhuysen S, Mandelstam S, Suls A, Audenaert D, Deconinck T, Claes LR, Deprez L, Smets K, Hristova D, Yordanova I, et al. (2012) KCNQ2 encephalopathy: emerging phenotype of a neonatal epileptic encephalopathy. Ann Neurol 71:15-25.

Zhang H, Craciun LC, Mirshahi T, Rohács T, Lopes CMB, Jin T, and Logothetis DE (2003) PIP(2) activates KCNQ channels, and its hydrolysis underlies receptormediated inhibition of M currents. Neuron 37:963-975.

Address correspondence to: Harley T. Kurata, Department of Pharmacology, Alberta Diabetes Institute, University of Alberta, 9-70 Medical Sciences Bldg., Edmonton, AB T6G 2H7, Canada. E-mail: kurata@ualberta.ca 